\title{
Romper con el paternalismo, el empoderamiento de las personas
}

\author{
Maria Martinez Lobo'; Miryam Alonso Galilea ${ }^{2}$
}

Recibido: 31/03/2021 / Aprobado: 18/05/2021

Resumen. En este artículo se describe lo que es empoderamiento versus paternalismo. Las diferentes definiciones y acepciones para el mismo término. Posteriormente se analizan las escalas de evaluación, destacando la Escala de Agencia Personal y Empoderamiento (ESAGE) que mide la autoeficacia, autonomía, autodeterminación y control. En cuanto a la metodología se analiza en distintos niveles de análisis: individual, grupal, organizacional y comunitario. Se analiza el empoderamiento como un proceso social multidimensional para la potenciación a nivel psicológico, nivel organizacional y nivel comunitario. El artículo finaliza, con el ejemplo de un caso práctico, una mujer con trastorno mental grave y su proceso de empoderamiento y potenciación.

Palabras clave: Empoderamiento, protagonista, paternalismo, comunitario, mujer empoderada.

\section{[en] Breaking with paternalism, empowerment of the people}

\begin{abstract}
This article describes what empowerment versus paternalism is. The different definitions and meanings for the same term. Subsequently, the evaluation scales are analyzed, highlighting the Personal Agency and Empowerment Scale (ESAGE), which measures self-efficacy, autonomy, self-determination and control. Regarding the methodology, it is analyzed at different levels of analysis: individual, group, organizational and community. It is therefore a multidimensional social process for empowerment at the psychological, organizational and community levels. The article ends, with the example of a practical case, a woman with a severe mental disorder and her empowerment and empowerment process.
\end{abstract}

Keywords: Empowerment, protagonist, paternalism, community, empowered woman.

Sumario. 1. Introducción: Empoderamiento versus paternalismo. 2. Metodología. 3. Evaluación. 4. Desarrollo de la Intervención. 4.1. Nivel psicológico. 4.2. Nivel organizacional. 4.3. Nivel comunitario. 5. Conclusiones. 6. Ejemplo práctico. Documentos. Bibliografía.

Cómo citar: Martinez Lobo, M.; Alonso Galilea, M. (2021): Romper con el paternalismo, el empoderamiento de las personas, en Cuadernos de Gobierno y Administración Pública 8-1, 41-53.

\section{Introducción: Empoderamiento Vs Paternalismo}

El término empoderamiento humano empoderar (se), es una traducción del inglés to empower, que se emplea en textos de sociología política con el sentido de "conceder poder a un colectivo desfavorecido socioeconómicamente para que, mediante su autogestión, mejore sus condiciones de vida". Puede usarse también como pronominal: «Se trata pues de empoderarnos, de utilizar los bienes y derechos conseguidos, necesarios para el desarrollo de los intereses propios» (Alborch Malas, 2002).

Por lo tanto ¿Qué es el empowerment? ¿Qué significa este constructo? Se trata de un concepto de difícil tra- ducción, y que no capta en su traducción al castellano la significación completa que Rappaport le confiere. Literalmente puede ser traducido como empoderar, es decir, dar poder, dotar de poder, pero el término empowerment se traduce generalmente, no cómo empoderar sino como potenciación o fortalecimiento.

De forma intuitiva, bajo el concepto de potenciación caben otros conceptos e ideas como el de autonomía, autogestión, pensamiento crítico e, incluso, libertad.

No hace mucho el término empoderamiento era desconocido para la mayoría de las personas. En intervención social, este concepto, puede ser entendido como "proceso por el cual las personas fortalecen sus capaci-

Terapeuta Ocupacional

mmartínezto@hotmail.com

2 Directora/Psicóloga. Centro de Rehabilitación Laboral de Getafe

miryamalonso@gmail.com 
dades, confianza, visión y protagonismo como grupo social para impulsar cambios positivos de las situaciones que viven" (Murguialday y otros, 2001).

Tuvo su origen en las estrategias de la Educación Popular, desarrolladas sobre todo por el educador brasileño Paulo Freire en los años sesenta. Según esta corriente, la educación es un proceso participativo, en el que el aprendizaje se basa en la experiencia práctica de las propias personas y grupos. De hecho, Rowlands (1995) señala que hay una gran similitud entre la noción de empoderamiento y las ideas defendidas por Freire, puesto que los individuos empoderados se convierten en "sujetos" de sus propias vidas y desarrollan una "conciencia crítica" -es decir, comprenden su entorno social, lo que les conduce a la acción. No es malo empoderarse porque el empoderamiento parte de la alfabetización y desde el conocimiento crítico.

Como puede observarse continuamente pasamos de una visión individual, a una grupal y/ o social.

A partir de los años setenta, esta idea de empoderamiento empezó a ganar fuerza en las organizaciones populares, entre las cuales los movimientos feministas han profundizado su aplicación en teorías sobre la importancia de empoderar a las mujeres para el logro de una sociedad más igualitaria. El concepto fue propuesto por primera vez a mediados de los años 1980 por DAWN, una red de grupos de mujeres e investigadoras del Norte y del Sur, para referirse al "proceso por el cual las mujeres acceden al control de los recursos (materiales y simbólicos) y refuerzan sus capacidades y protagonismo en todos los ámbitos". Jain fue la economista india fundadora de DAWN. Desde este enfoque más feminista, el empoderamiento de las mujeres abarca desde el cambio individual a la acción colectiva, e implica la alteración radical de las estructuras y procesos que reproducen la posición subordinada de las mujeres como género. El empoderamiento, implica también tener acceso a los recursos, tanto materiales como simbólicos, lo que supone que es necesario lograr el empoderamiento económico.

No obstante, el término se consagrará definitivamente en 1995, durante la Conferencia Mundial de las Mujeres en Pekín convocada por Naciones Unidas, cuando se lo empleó por primera vez en los debates políticos como estrategia fundamental para que las mujeres asumieran un papel de liderazgo, con el fin de producir cambios que les permitieran participar en las mismas condiciones que los hombres en todas las esferas sociales y, particularmente, en la toma de decisiones. Dicho concepto figura en el texto final de la Declaración y de la Plataforma para la Acción, aprobada por unanimidad durante el evento.

Durante los últimos años el término "empoderamiento" ha ampliado su campo de aplicación, ha pasado a designar también al conjunto de colectivos vulnerables. La extensión de su utilización en los estudios incluye el desarrollo, el trabajo comunitario y social, y la cooperación para el desarrollo. Mientras, la visión de los grupos de mujeres y otros movimientos sociales va más allá. El empoderamiento sería "un proceso que propicia que las mujeres y otros grupos marginados incre- menten sus posibilidades, esto es, que accedan al uso y control de los recursos materiales y simbólicos, que ganen influencia y prestigio, y que participen en el cambio social. Estos cambios pragmáticos traen consigo también un proceso por el que las personas se conciencien de sus propios derechos, capacidades e intereses, y de cómo éstos se relacionan con los intereses de otras personas, con el fin de participar desde una posición más sólida en la toma de decisiones, y estar en condiciones de influir en ellas.

Por lo tanto, el término empoderamiento se aplica a todos los grupos vulnerables en un proceso por el cual las personas fortalecen sus capacidades, confianza, tienen visión y protagonismo como grupo social para impulsar cambios positivos de las situaciones que viven.

Los profesionales que intervenimos con personas en el ámbito de lo social, en mi opinión, debemos reaprender a preguntar: ¿Qué necesidades tiene la persona atendida ¿qué expectativas? ¿Qué motivaciones? ¿Qué es verdaderamente lo que demanda de nuestra atención? etc. y trabajar desde ahí. Preguntar, escuchar para saber cómo están las personas, que demandas nos hacen y así favorecer y fomentar su conciencia crítica.

La Organización Mundial de la Salud (OMS) considera que el "empoderamiento" es un concepto esencial de la promoción de la salud. La Declaración de AlmaAta y la Carta de Ottawa para la Promoción de la Salud reconocen su importancia para la prevención (de la enfermedad) y la promoción de la salud. Uno de los seis puntos clave para la guía de actuaciones que aparecen en la Estrategia Europea de Prevención y Control de las Enfermedades No Transmisibles establece que "las personas deben ser empoderadas para promocionar su propia salud, para interactuar con los servicios sanitarios y participar activamente en la gestión de la enfermedad". La Declaración de Salud Mental para Europa, el Plan de Acción en Salud Mental para Europa y el Pacto Europeo para la Salud Mental y el Bienestar reconoce que el empoderamiento de las personas con problemas de salud mental y de sus cuidadores es prioritario en las próximas décadas.

En promoción de la salud, el empoderamiento para la salud es un proceso mediante el cual las personas adquieren un mayor control sobre las decisiones y acciones que afectan a su salud. Se establece una distinción entre el empoderamiento para la salud del individuo y el de la comunidad. El empoderamiento para la salud individual se refiere principalmente a la capacidad del individuo para tomar decisiones y ejercer control sobre su vida personal. El empoderamiento para la salud de la comunidad supone que los individuos actúen colectivamente con el fin de conseguir una mayor influencia y control sobre los determinantes de la salud y la calidad de vida de su comunidad, siendo éste un importante objetivo de la acción comunitaria para la salud. Generalmente implica, en el beneficiario, el desarrollo de una confianza en sus propias capacidades y acciones, junto con el acceso al control de los recursos, la representación en los cuerpos de toma de decisiones y la participación de los procesos de planificación. 
Por otro lado, el concepto de empoderamiento ha ocupado un lugar primordial en la psicología comunitaria, denotando a la vez una orientación valórica y un proceso con componentes cognitivos, afectivos y conductuales (Rappaport, 1981; Zimmerman, 2000). На sido señalado como una de las vías fundamentales para el desarrollo y la transformación de las comunidades (Montero, 2003).

Para Powell (1990), el empowerment es el proceso por el cual los individuos, grupos y comunidades llegan a tener la capacidad de controlar sus circunstancias y de alcanzar sus propios objetivos, luchando por la maximización de la calidad en sus vidas. Supongamos que cinco personas de una comunidad local se muestran preocupadas por el vertido de desechos tóxicos que realiza una empresa de productos químicos en las inmediaciones de su comunidad. Estas personas se reúnen y deciden unirse para encontrar una solución a este problema. Supongamos, pues, que se organizan y crean en su comunidad local, una asociación para la defensa de su medio ambiente, y que consiguen progresivamente que otros miembros de la comunidad participen en la causa. La situación descrita representa un proceso potenciador que no sólo asume un nivel individual (originalmente procedente de individuos -vecinos- con sentido de control personal, con conocimiento crítico de la realidad sociopolítica) y grupal (unión de estos individuos reflejado en la creación de una organización en la comunidad) sino comunitario (implicación de otros miembros en la asociación). Supongamos que esta asociación moviliza otros recursos de su propia comunidad, como, por ejemplo, buscar y conseguir el apoyo de otras estructuras mediadoras de su comunidad - asociación de vecinos, escuela local, sindicato político-. Apoyada por otras estructuras de la comunidad supongamos finalmente que esta fuerza local consiga modificar la política local en materia de vertidos de desechos. En este caso hipotético, individuos, organización y comunidad han movilizado recursos que les ha permitido tener la capacidad de controlar sus circunstancias y de alcanzar sus propios objetivos, luchando por optimizar su calidad en sus vidas. En definitiva, desde distintos niveles, y en específicamente desde un nivel individual a uno comunitario, se ha producido un proceso de potenciación. Esto es, en síntesis, lo que podría ser un proceso de empowerment.

McWhirter (1995), describe el empoderamiento de manera más detallada. El proceso por el que las personas, las organizaciones o los grupos carentes de poder:

a) toman conciencia de las dinámicas del poder que operan en su contexto vital,

b) desarrollan las habilidades y la capacidad necesaria para lograr un control razonable sobre sus vidas,

c) ejercitan ese control sin infringir los derechos de otros

d) apoyan el empoderamiento de otros en la comunidad.

Por tanto, teniendo en cuenta todas estas definiciones, podemos decir a grandes rasgos que el empodera- miento permite que las personas tomen consciencia de la situación en la cual viven y que, a partir de esta percepción sobre su contexto, puedan desarrollar su capacidad para cambiarlo, es decir, participen activamente en el proceso de transformación.

\section{Metodología}

A partir de estas ideas, podemos considerar que el empowerment, la potenciación puede producirse en distintos niveles de análisis: individual, grupal, organizacional y comunitario. Es, por tanto, un constructo multinivel; es necesario analizar la realidad de las personas en función de los diferentes niveles para comprender por qué ciertos aspectos organizacionales, políticos o económicos tienen un peso específico para adquirir, o en contraposición, para inhibir, los procesos de control y de dominio (empowerment).

Tanto los procesos como los resultados de la potenciación varían a lo largo de los diferentes niveles sugeridos por la teoría del empowerment. Así Zimmerman (2000) establece una comparación entre los procesos y resultados que operan a nivel individual, organizacional y comunitario. Este autor considera como procesos de potenciación en el ámbito individual el aprender a tomar decisiones, a manejar recursos, o trabajar en equipo con otras personas. El resultado operativo de una potenciación en el ámbito individual, puede ser, según apunta este autor, el sentimiento de control personal, la conciencia crítica o el comportamiento participativo.

$\mathrm{Y}$ en el otro polo, el concepto de paternalismo y cómo nos ocupa en nuestro trabajo de intervención social. El paternalismo, según la Real Academia Española es la tendencia a aplicar las formas de autoridad y protección propias del padre en la familia tradicional a relaciones sociales de otro tipo; políticas, laborales, etc. Así, en nuestra ocupación social, el paternalismo consiste en recurrir al poder o a la autoridad que proceden de la profesión para poder intervenir en favor de las personas atendidas y de sus intereses, pudiendo llegar en cierto modo a restringir su autonomía y su capacidad de decisión.

Los-as profesionales cuando atendemos a las personas, nos movemos entre el código deontológico de nuestras respectivas profesiones, el marco teórico y el compromiso ético en nuestras actuaciones.

Podemos diferenciar el paternalismo negativo del positivo. El primero, referido a no tener en cuenta la opinión de la persona atendida y tomar decisiones por ella; y el segundo, centrado en priorizar el bienestar de la persona atendida, su autonomía en los supuestos indicados por él. Los seres humanos somos personas adultas, nunca nadie puede tomar decisiones por los ciudadanos, aunque en ocasiones hay excepciones que se pueden explicar desde la filosofía política y desde la ética. Por ejemplo, "es conveniente que tome la medicación por mi bien y mi estabilidad clínica ya que si no lo hago puedo hacerme daño a los demás". En este caso, proteger a la persona por su bien es distinto del autoritarismo. 
Para ello, la elección del lenguaje es fundamental. El lenguaje que se emplea tradicionalmente para describir los procesos de ayuda, potencia inconscientemente la dependencia de las personas, y crea una visión en la que las personas son clientes que precisan de una ayuda de naturaleza unidireccional. Este lenguaje según Rappaport, limita el descubrimiento de recursos y potencialidades y reduce la posibilidad de que las personas se ayuden entre sí. Un acercamiento al enfoque del empowerment sustituye términos como cliente y experto por participante y colaborador. En definitiva, se debe optar por un lenguaje que transmita a los sujetos la oportunidad que tienen para potenciar sus propias habilidades y para controlar/ movilizar sus propios recursos, prescindiendo totalmente de expresiones en las que los sujetos perciban que son objeto de ayuda o que se les va a proporcionar servicios.

Entonces ¿cómo debemos actuar los/as profesionales de lo social? Como ya sabemos los extremos no son buenos. El no paternalismo sería el comportamiento ético por excelencia que deberíamos desarrollar; velando por el bienestar de la persona, teniendo en cuenta su opinión, su autodeterminación acompañando siempre hacia su autonomía en la sociedad. Por ejemplo, una persona solicita ayuda alimentaria o ayuda para el transporte y sin reflexionar por qué no puede llegar cubrir esa necesidad; qué está fallando y qué puede hacer para mejorarlo, los profesionales aceptan ese apoyo sin ir más allá y proponer también otro tipo de intervenciones que ayuden realmente a la persona. Moviéndonos en los extremos, muchas veces me pregunto: si la salida del paternalismo es el liberal abandono ¿qué escogería?

Hay algunas formas de paternalismo "más sutiles" que se pueden dar a menudo en las atenciones a personas, como es el caso de no informar sobre determinados temas a la persona atendida o el hecho de revelar información que debería ser confidencial "por el bien del mismo", o por ejemplo tratar a las personas con discapacidad como si fuesen "chicos y chicas" toda la vida, siendo muchas veces personas con más edad que los propios profesionales, otra vez con el lenguaje discriminatorio.

En este sentido, si como he comentado antes el paternalismo rompe con la autonomía de la persona atendida que a su vez debería estar garantizada, es necesario preguntarse: ¿está justificado el paternalismo en ciertas situaciones? Algunas voces de expertos que han reflexionado y debatido sobre este tema, piensan que en algunas circunstancias como en el caso de los niños-as, de personas con dificultades en alguna de sus capacidades, de personas con crisis psiquiátricas graves, etc. el paternalismo está totalmente justificado y que incluso, sería amoral recurrir al valor de la autonomía. Aun así, en estas ocasiones habría que respetar la autonomía orientándola hacia los tutores, familiares de estas personas o bien documentos donde la persona deja por escrito cómo actuar con ella en caso de crisis (por ej.: Protocolo de actuación ante el paciente con enfermedad mental en crisis grave con riesgo de autolesión o de suicidio, etc.)

Para tratar de entender la complejidad de lo que nos ocupa, voy a plantear un dilema sobre la libertad y la au- tonomía de las personas. Para ello, podemos preguntarnos ¿habría alguna situación donde estaría, por ejemplo, aceptada únicamente una acción coercitiva en personas con una descompensación psiquiátrica?

Joan Caminas, Doctor en Filosofía, en su conferencia "Derechos humanos y Éticas de los cuidados "en VII Congreso de la Asociación Madrileña de Rehabilitación Psicosocial 2020: "Cartografiando los Derechos Humanos en Rehabilitación Psicosocial”, nos explica cómo en ocasiones aplicar una medida coercitiva puede ser por tu bien. Pues bien, basándonos en la Filosofía de la ética existen unas acciones coercitivas protectoras de primer nivel, que serían adecuadas. Para aceptar esto que parece inasumible desde la autonomía y el empoderamiento, ¿qué condiciones deben cumplirse?:

1. la acción que vamos a llevar a cabo, en este caso la medida de coerción debe ser necesaria para proteger un bien básico, en este caso la vida de la persona. Debe ser efectiva, eficiente, proporcionada y respetuosa es decir ¿hemos agotado todas las medidas de ayuda anteriores? ¿ya no podemos hacer nada más?

2. la persona, en ese momento, no tiene capacidad cognitiva y volitiva; o al menos, no es capaz de entender esa situación.

3. si tuviera la capacidad de entenderlo, estaría de acuerdo con la acción (documento de voluntades anticipadas).

4. no se vulnera un derecho fundamental a terceras personas.

5. genera un "malestar moral" razonable en las personas que lo deciden y ejercen. Es decir, es una medida correcta éticamente, pero me hace sentir mal, algo así como me produce "remordimientos de conciencia".

Sin duda, un tema muy polémico y discutible. Sin embargo, desde los modelos teóricos, por ejemplo, el modelo centrado en la persona, o el modelo de recuperación, en el caso de personas con trastorno mental grave y los códigos deontológicos y éticos, estos dilemas pueden resolverse.

\section{Evaluación}

En la experiencia empírica de diseñar, implementar y evaluar programas de desarrollo humano con poblaciones marginadas y/o vulnerables, se ha observado que éstos van más allá de cambios en conductas, logrando incluso el desarrollo de "agencia personal" y empoderamiento.

El Instituto Mexicano de Investigación de Familia y Población, A.C. (IMIFAP) ha estado involucrado durante más de 20 años en el desarrollo de programas de desarrollo humano y comunitario, basados en la investigación en torno a temas de salud y disminución de la pobreza.

En el punto anterior ya hemos explicado qué es empoderamiento. Pero ¿qué es agencia? En psicología, 
la agencia se ha definido como "el grado de funcionamiento autónomo" (Kagitcibasi, 2005), añadiendo que la agencia es el control deliberado que subyace la autonomía. Según el Premio Nobel de Economía, Amartya Sen, la agencia es la habilidad de definir las metas propias de forma autónoma y de actuar a partir de las mismas: "aquello que una persona tiene la libertad de hacer y lograr en búsqueda de las metas o valores que él o ella considere importantes" (Sen, 1985). Por ende, el concepto de agencia incorpora más que la acción; también incluye la intención, el significado, la motivación y el propósito que los individuos imprimen a sus actividades (Kabeer; Sen, 1999).

Proponemos usar el término agencia como un concepto abarcador (paraguas) que capture los diversos aspectos de un funcionamiento individual saludable y competente.

El Cuestionario para la Evaluación de la Discapacidad de la Organización Mundial de la Salud (WHODAS 2.0, por sus siglas en inglés) es un instrumento de evaluación genérico desarrollado por la OMS para brindar un método estandarizado de medición de la salud y la discapacidad entre las culturas. Fue desarrollado a partir de un conjunto integral de dimensiones de la Clasificación Internacional del Funcionamiento, la Discapacidad y la Salud (CIF), por sus siglas en inglés) que son lo suficientemente confiables y sensibles para medir la diferencia generada por una intervención determinada. Esto se logra al evaluar al mismo individuo antes y después de la intervención. Se utilizaron una serie de estudios de campo sistemáticos para determinar la aplicabilidad intercultural, la confiabilidad y la validez del cuestionario, así como también su utilidad en investigaciones sobre los servicios de salud. Se encontró que WHODAS 2.0 es útil para la evaluación de los niveles de salud y discapacidad en la población general mediante encuestas y para la medición de la efectividad clínica y de los aumentos de la productividad originados por las intervenciones.

El concepto Agencia y los conceptos psicológicos equivalentes son procesos que ocurren en el nivel individual. No obstante, el proceso de desarrollar agencia usualmente tiene implicaciones a nivel social. Una vez que la agencia comienza a impactar en la familia, los colegas, las organizaciones y la comunidad, tal y como ya hemos comentado se convierte en empoderamiento.

Los siguientes son conceptos psicológicos que se incluyeron en la construcción de la Escala Para Medir Agencia Personal y Empoderamiento (ESAGE):

1. Autoeficacia: Bandura ha vinculado la idea de agencia con la autoeficacia, proponiendo que los individuos poseen un sistema propio que les permite ejercer cierto grado de control sobre sus pensamientos, sentimientos y acciones (Bandura, 1998, 2001, 1996).

2. Autonomía: Kagitcibasi define la agencia y la autonomía como coincidentes: "Autonomía es ser agente y al mismo tiempo actuar con voluntad propia, sin un sentido de coerción" (2005, p. 404). Se ha encontrado que la autonomía está vinculada con relaciones satisfactorias y auténticas con los otros (Hodgins,
Koestner, \& Duncan, 1996) y con el bienestar (Ryan \& Deci, 2000), tanto en comunidades de tipo más tradicional como de tipo más occidental (Deci \& Ryan, 2000).

3. Autodeterminación: la teoría de autodeterminación propone que la verdadera agencia requiere de la autonomía, afirmando que la competencia (adquirida a través de habilidades), la conectividad y la autonomía son esenciales para el crecimiento personal, la integridad y el bienestar duraderos (Deci \& Ryan, 2000).

4. Control: el concepto de control a menudo se asocia con el "Locus de Control", un continuo propuesto por Rotter, 1966 el cual refleja las expectativas generalizadas de un individuo acerca de lo que determina las recompensas (o la falta de recompensas) que uno obtiene en la vida. La gente que tiene un locus de control interno fuerte, cree que sus propias acciones determinan lo que le ocurre; el éxito o el fracaso se debe a sus propios esfuerzos. En contraste, el control externo implica que la conducta propia no importa tanto y que las recompensas en la vida son controladas por la suerte, el azar u otras personas que son poderosas. Por lo tanto, la gente con un alto control externo ve poco impacto de sus propios esfuerzos en la cantidad de información que recibe.

Con el fin de poder medir estos dos componentes, agencia y empoderamiento se construyó una escala que muestra que son dos conceptos relacionados y separados a la vez: la Escala de Agencia Personal y Empoderamiento (ESAGE) está conformada por dos subescalas; agencia personal con 35 reactivos y empoderamiento con 7.

La agencia es difícil de medir porque a menudo opera de distintas maneras; puede tomar la forma de toma de decisiones, negociación, asertividad y otros procesos cognitivos de reflexión y análisis (Kabeer, 1999). En la literatura sobre psicología, la agencia frecuentemente se vincula con diversos conceptos psicológicos similarmente "nombrados" (autoeficacia, autonomía, control, autodeterminación, etc.) y se mide a través de ellos.

En un primer esfuerzo de medición de la agencia y el empoderamiento de las personas, se diseñó la Escala de Agencia Personal y Empoderamiento (ESAGE) que incorporó reactivos agrupados en las siguientes variables:

1. Auto-eficacia.

2. Autodeterminación.

3. Control sobre mis conductas

4. Pensamiento independiente

5. Identificación de necesidad de cambio

6. Miedo al éxito

7. Reconocimiento de mi aprendizaje

8. Percepción de mi contexto

9. Control sobre mi entorno.

La ESAGE es auto-aplicable y cada reactivo puede ser respondido en una escala tipo Likert de frecuencia con cuatro opciones: "nunca", "casi nunca", "casi siempre", "siempre". 
A pesar de la dificultad de medir el empoderamiento, la medición puede ser importante, como señala Kabeer, el intento por cuantificar el concepto de empoderamiento parece colocar la idea en un "terreno más sólido y objetivamente verificable" (Kabeer, 1999).

Existen muchas investigaciones en el área del empoderamiento de la mujer, y hay una serie de enfoques distintos para su medición. La Conferencia del Cairo de 1994 vinculó el empoderamiento con un amplio grupo de resultados demográficos favorables y estableció tres áreas clave que conciernen al empoderamiento de la mujer:

- mejor salud.

- acceso a recursos económicos.

- y reducción en la desigualdad de género (Williams, 2005).

Los economistas del desarrollo demuestran el vínculo entre el empoderamiento de la mujer y el crecimiento económico, fallas de mercado, insumos laborales, etc.

Por otro lado, se han emprendido muchas iniciativas para mejorar el empoderamiento de las personas con discapacidad en los países en desarrollo. Para ello, se realizó una revisión sistemática de la literatura. Los artículos eran elegibles cuando describían el desarrollo, la validación, la traducción o el uso de un instrumento que mide el empoderamiento en el contexto de la discapacidad. Los instrumentos fueron evaluados por sus propiedades psicométricas y sus propiedades de equivalencia cuando fueron traducidos. Los resultados obtenidos fueron 36 artículos en los que se desarrollaron, validaron, tradujeron o utilizaron 17 cuestionarios. Los cuestionarios variaron en el constructor de empoderamiento, la población objetivo y las propiedades psicométricas. Ninguno de los cuestionarios fue elaborado o validado en un país en desarrollo. Las propiedades psicométricas y los criterios de equivalencia no se describieron ni midieron adecuadamente en ningún artículo. La Empowerment Scale (ES) de Rogers fue el cuestionario validado, traducido y utilizado con mayor frecuencia, recibiendo el mayor número de calificaciones positivas para las propiedades psicométricas. Por lo tanto, el EE es la herramienta más utilizada para medir el empoderamiento, pero aún falta una validación adecuada en el contexto de un país en desarrollo. La validez cultural debe evaluarse en cualquier cultura antes de su uso. Se necesitan más investigaciones para desarrollar instrumentos de empoderamiento para los países en desarrollo y evaluar los criterios de equivalencia, incluidas las propiedades psicométricas de dichos cuestionarios.

\section{Desarrollo de la intervención}

Hasta el momento, hemos tratado de argumentar los motivos por los que el empoderamiento se convierte en nuestro punto de partida para encaminar y adecuar la intervención a las personas que atendemos, acompañándolos en procesos de crecimiento personal, independientemente del colectivo social al que pertenezcan.

El empoderamiento tal y como hemos señalado, es un proceso social multidimensional por lo tanto debemos comprenderlo a nivel individual y poblacional, de esta manera tanto los individuos como los grupos a los que pertenecen, conseguirán un mejor conocimiento y mayor control sobre sus vidas y desde aquí transformar su entorno social y político para mejorar sus circunstancias vitales que están relacionadas con la salud.

Por ello y rescatando el concepto de empowerment de María Isabel Hombrados y Juan Manuel Domínguez, que aportan en "Estrategias de Intervención Psicosocial", la "Teoría de la Potenciación" donde explican el proceso por el cual el individuo o la comunidad de individuos, adquiere recursos y control para asumir actividades o implicarse en conductas que previamente trascendían sus habilidades (Adams, 1990). Por ejemplo, una persona inmigrante tendrá mayor facilidad en su proceso de potenciación, si se le da acceso a una red de recursos, conocimiento del idioma, trabajo, etc.

Además, cuando se habla de potenciación se hace a distintos niveles: las personas, las organizaciones y las comunidades. Pero en cada uno de estos niveles se producen dos hechos diferenciados: potenciar y ser potenciado.

En definitiva, el empoderamiento es el resultado del desarrollo de la potenciación del individuo, dónde nos centramos en crear las condiciones más adecuadas para cubrir las necesidades específicas de un grupo, con características similares.

En nuestra intervención, debemos tener en cuenta la existencia de niveles de análisis interdependientes para la potenciación (Zimmerman 1995, 2000):

- Nivel psicológico: nivel de análisis individual.

- Nivel organizacional: procesos que proporcionan el apoyo mutuo necesario.

- Nivel comunitario: se refiere al trabajo de los individuos conjuntamente con el fin de mejorar la calidad de vida en comunidad.

Tras observar esta correlación implícita entre el individuo y el grupo de individuos, nos disponemos a ofrecer estas modalidades de intervención:

\subsection{Nivel psicológico}

Para cada uno de nosotros, el empoderamiento es un elemento importante del desarrollo humano. Es un proceso de toma de control sobre su propia vida y responsabilidad de las actuaciones que tienen como propósito alcanzar la totalidad de su capacidad.

Habitualmente podemos encontrar cuatro dimensiones en el empoderamiento:

- autoconfianza,

- participación en las decisiones,

- dignidad y respeto,

- pertenencia y contribución a una sociedad más plural.

Dicho proceso comienza por la definición individual de las necesidades y objetivos deseados centrándose en el desarrollo de las capacidades y recursos que lo apo- 
yen. El empoderamiento de los individuos está dirigido a ayudar a la autodeterminación y autonomía, para que pueda ejercer más influencia en la toma de decisiones sociales y políticas, y para aumentar su autoestima.

Como resultado del proceso de empoderamiento emerge en el individuo una "conciencia crítica", en tanto se vuelve crítico de las normas sociales que había aceptado previamente sin cuestionar (Kabeer, 1999). Conforme las personas desarrollan la capacidad y adquieren los medios necesarios para elegir, comienzan a establecer valores y preferencias personales que pueden desafiar las normas sociales tradicionales.

En las diferentes intervenciones sociales, es muy importante el modelo teórico en el que nos basamos como profesionales. Por ejemplo, según el Modelo de Atención Centrado en la Persona, de Salud Mental de Intress (MASMI 2.0) cada persona tiene el derecho y la capacidad de elegir por sí misma su manera de obrar, de responder de sus actos y de ser dueña de escoger su propio destino, obrando con libre determinación y autonomía. La perspectiva del modelo de recuperación, toma a la persona como la verdadera protagonista de su proceso terapéutico y de crecimiento. Es el actor de su vida. En los diferentes Encuentros (expertos en primera persona y profesionales) que hemos tenido para elaborar el MASMI de Intress, hemos trabajado el concepto "paciente empoderado y altamente implicado", estableciendo una serie de conclusiones en cuanto a la persona atendida y el profesional:

- Los profesionales y las personas en atención podemos colaborar para establecer un modelo renovado de intervención en salud mental en Intress, basado en la colaboración y el trato humano, un liderazgo compartido que contribuya a la transformación de la atención en salud mental.

- La flexibilidad, la horizontalidad, la humanización de la atención, el conocimiento por experiencia, el apoyo mutuo, la comunicación bidireccional, se ven como factores claves para alimentar este renovado modelo.

- Un modelo que se enfrenta a resultados ambiciosos con cotas altas separadas de la remisión de síntomas o de la mera ocupación, destinados a alcanzar y desarrollar la maestría personal y el crecimiento como persona.

- Para enfrentarse a estos nuevos retos se hace imprescindible evolucionar hacia una atención participativa dentro de un ecosistema multifactorial en el que están involucrados diferentes actores, agentes, personas y en particular la propia persona en atención adquiriendo un papel activo no solo en su proceso de cambio personal sino en los procesos de atención a desarrollar en lo servicios.

- Un modelo que establece sus coordenadas en valores guía como la esperanza, la autodeterminación, la empatía y la capacidad de superación.

Otra referencia bibliográfica y ejemplo desde la Salud Mental, la encontramos en Guía PARTISAM, don- de ofrecen una serie de pautas en los procesos individuales de intervención y tratamiento:

- Escucha activa del/de la profesional: la persona necesita transmitir esa experiencia para ser comprendido y afrontar la enfermedad.

- Reconocer a la persona como experta en salud mental: el profesional debe cambiar la visión sobre la persona para que su papel pase a ser activo por dar valor a su experiencia.

- Garantizar las decisiones informadas: la persona debe tener acceso al Plan Individualizado de Atención (en algunos centros participan durante todo el proceso y en las reuniones donde se establece el plan de objetivos)

- Promover la vida independiente: aceptando la situación actual en la que se encuentra la persona y dotar de herramientas que faciliten el acceso a la participación.

- Fomentar la construcción de un proyecto de vida: y para poder llevarlo a cabo es necesaria la inclusión económica y social, donde la persona tenga un trabajo, educación, ocupación en movimientos asociativos, mejora en los roles familiares y sociales.

- Favorecer la autodeterminación

- Diseñar un "Plan de Crisis Acordado": se negocian los términos ante situaciones hipotéticas futuras de crisis, especificando unas voluntades anticipadas. De esta manera, puede llegar a reducirse el número de ingresos hospitalarios.

- Establecer planes individualizados de recuperación personal.

- Implicarse en la elección de sus cuidadores/educadores.

En todo momento, nuestra intervención se centrará en las posibilidades y capacidades personales, con enfoque positivista y optimista en la persona a la que ofrecemos una relación de ayuda. No perderemos de vista, el respeto por el otro, su capacidad de decisión, su perspectiva y la libertad de elección. Esto hará que tenga mayor iniciativa y sea activa en su propio proceso de recuperación, interactuando y transformando su entorno según sus necesidades y aspiraciones, transformándose a su vez, a sí mismos.

La persona es la protagonista y el agente de su propio cambio.

Las comunidades juegan un papel importante en la cohesión entre individuos. Pueden llegar a generar una red social de ayuda interesante, durante periodos de vulnerabilidad. Además, el empoderamiento de una comunidad, trae consigo el empoderamiento individual pues comprende el sentimiento de pertenencia a la comunidad, el desarrollo de actividades políticas y la participación en ellas, el liderazgo en el proceso de toma de decisiones y el acceso a los recursos.

"Recuperar la salud mental es mucho más que recobrarse de la enfermedad, conlleva la recuperación del estigma, de la falta de recursos, de las contrariedades de la vida e incluso de la pobreza y otras dificultades, 
de la desesperanza asociada a múltiples fracasos, etc., asimismo implica también superar el trauma inherente a sufrir un trastorno mental grave o recibir tratamiento psiquiátrico.” Vázquez y col. (2010)

\subsection{Nivel organizacional}

En este punto nos queremos referir a las intervenciones que llevamos a cabo desde el punto de vista grupal, lo colectivo. En este sentido, todo el movimiento de asociaciones tanto de familiares como en primeras personas de cualquier colectivo, nos servirá como ejemplo de intervención.

En el ámbito de salud mental, ha surgido un movimiento de cambio en estos últimos años encaminado a este tipo de intervención, basada en el empoderamiento de las personas con problemática emocional, los Grupos de Ayuda Mutua, más conocidos como GAM.

Surgen de la idea de que toda persona, con sus habilidades y su experiencia personal, tiene una valiosa capacidad para ayudar a los demás y por extensión, ayudarse a sí misma; las personas que participan en él lo hacen de forma voluntaria y generalmente, en ausencia de un profesional, aunque pueden solicitar puntualmente esta ayuda. Son personas que comparten un mismo problema que se comparte y se construye de una manera más objetiva y menos emocional pudiendo ser menos emocional, dada que la situación es vista "desde fuera".

Conlleva una situación horizontal, con formas relativamente democráticas, pacíficas y concertadas que actúan como una empresa conjunta. Se rigen según una serie de conceptos/principios:

- Autogestión.

- Autogobierno.

- Autoadministración.

- Definición autónoma de objetivos y de organización.

- Autofinanciamiento.

Una petición recurrente en los debates abiertos es el reconocimiento de las personas con problemas de salud mental como expertas, por su conocimiento de primera mano de sus propias necesidades y recursos para atenderlas. Este planteamiento/reconocimiento permitiría tomar el control de sus propias vidas, enriquecer el proceso de recuperación y, por lo tanto, avanzar hacia la plena autonomía.

Además del reconocimiento de derechos, es otro de los elementos que ha caracterizado al movimiento asociativo en el ámbito de salud mental es su lucha incansable contra el estigma social y la discriminación.

Resulta sencillo localizar las ventajas de conformar asociaciones o intervenir con las personas con problemas de salud mental, teniendo en cuenta los valores de la potenciación y el empoderamiento, pero a la contra, surgen barreras que dificultan estas vías de actuación.

Los grupos de usuarios-as y cuidadores han formulado varios atributos clave del empoderamiento, algunos de los cuales pueden conducir a recomendaciones de ac- tuación para el empoderamiento en diferentes niveles de personas atendidas:

- Poder para tomar decisiones: a veces, los profesionales de salud mental consideran que a las personas atendidas del servicio y sus familias les falta capacidad para tomar decisiones o para hacerlo correctamente. Como consecuencia los servicios adoptan, frecuentemente, una postura paternalista limitando el número o calidad de las decisiones que las personas atendidas y sus familias pueden tomar. Hay que resaltar que, sin apoyo en la toma de decisiones, las personas atendidas quedan atrapados en relaciones de dependencia que se prolongan en el tiempo. Nadie puede ser independiente si no tiene ocasión de tomar decisiones importantes sobre su vida. En algunos casos, la negación de la capacidad jurídica es el obstáculo principal para la toma de decisiones: se prohíbe legalmente que una persona tome decisiones. Las personas con problemas de salud mental deberían gozar de capacidad jurídica, en términos de igualdad, en todos los aspectos de la vida y asimismo los estados tienen la obligación de proporcionar ayuda a las personas que necesitan asistencia para tomar decisiones.

- Acceso a la información y a los recursos: las decisiones no se toman en vacío. Las personas sólo deciden correctamente cuando tienen la información suficiente para valorar las consecuencias que se derivan de varias posibilidades de elección, dicho con otras palabras: las decisiones tienen que ser informadas. De nuevo y como consecuencia del paternalismo, los profesionales de salud mental restringen frecuentemente la información creyendo que lo hacen en beneficio de las personas atendidas. Este hecho se convierte en una profecía cumplida, puesto que como falta la información adecuada las personas atendidas deciden de forma que confirma la creencia de los profesionales sobre su inadecuación para la toma decisiones.

- Abanico de opciones donde elegir: hacer una elección sensata significa que las personas atendidas han podido conocer todas las opciones pertinentes posibles, y que han recibido toda la ayuda requerida y adecuada para realizar dicha elección.

\subsection{Nivel comunitario}

En todos estos niveles y en su interrelación, no debemos perder de vista los principios de la intervención comunitaria:

- Características sociodemográficas y distintas necesidades.

- Las características del contexto (familia, trabajo, amigos) determinan el tipo de actuación.

- Cambios en el sentido de potenciación en función de las necesidades del sujeto y características del contexto.

- Interacción de tres componentes: intrapersonal (como se ven a sí mismos), interactivo (idea de su 
comunidad y control sobre ambiente) y comportamental (actuaciones encaminadas a la potenciación).

La práctica de la potenciación encuentra su vía de actuación a través de:

- La participación ciudadana: medio por el que las personas acceden y controlan los recursos, e implica tanto la toma de conciencia colectiva como el compromiso individual de las personas. Fomentan la conciencia de pertenencia a la comunidad.

- El desarrollo comunitario: cooperación voluntaria y ayuda mutua de las personas que conviven en un entorno similar. Asociaciones de vecinos como claves para el desarrollo comunitario.

Hombrados y Gómez proponen detectar necesidades, crear entidades que propicien la autoayuda, desarrollar oportunidades de empleo, promover actuaciones que sirvan para mejorar la salud física y mental, enseñarles a ser líderes de entidades dentro de la comunidad.

Fawet et al. señalan que la potenciación de la comunidad debe entenderse como resultado de la interacción entre los factores ambientales y comportamentales atendiendo a tres dimensiones: la persona o grupo, el ambiente y el nivel de potenciación. Se plantea como modelo transaccional en el que los elementos ambientales que afectan a las acciones son a la vez influidos por las acciones de los individuos y grupos:

- Factores personales o grupales.

- Factores ambientales.

- Estresores y barreras.

- Apoyo y recursos.

En este sentido, encontramos en la Comunidad de Madrid un ejemplo excelente de fortalecimiento organizacional y comunitario en el ámbito específico de la cultura. En esta comunidad, la Coordinadora de Asociaciones Culturales de Madrid (Coacum), creada en 1988, agrupa a diversos colectivos culturales y vecinales de barrios y municipios de la región madrileña. Se trata de una Federación de Asociaciones Culturales que tiene como finalidad el desarrollo sociocultural de la Comunidad de Madrid. Según sus propias palabras: orientan sus proyectos hacia una transformación social, por un concepto plural de la vida y del mundo, basado en una participación social más amplia que la que se lleva a cabo desde los poderes públicos y privados. Esta Coordinadora que apoya y fomenta la cultura participativa, ligada a los movimientos ciudadanos y asociaciones de vecinos, y que se convierte en un lugar de encuentro y de comunicación, propone el diálogo como motor de la actividad cultural, tanto en el ámbito artístico (teatro, pintura, música, cine, etc.) como en el social (debates, conferencias, coloquios, etc.). Coacum lleva a cabo las acciones siguientes:

- Coordina las actividades de las asociaciones miembros para una mayor eficacia, con lo que posibilita la organización de actividades o montajes que exceden los recursos de una sola.
- Busca la colaboración con la Administración y otros organismos para facilitar la tarea de estas asociaciones.

- Pone en relación a las asociaciones favoreciendo el intercambio de recursos materiales y humanos.

- Elaborar notas informativas y material documental (sobre centros culturales, Festivales, Muestras, etc. de la Comunidad de Madrid, ayuntamientos y de la Comunidad Europea);

- Gestiona igualmente proyectos y programas de trabajo con instituciones como el Centro de Documentación Teatral, la Sociedad General de Autores, etc.

Vemos, por lo tanto, que esta coordinadora cultural ayuda a crear en el ámbito de la cultura una comunidad fortalecida al ser una organización potenciadora y potenciada que trabaja con los recursos de la comunidad en interés de un proyecto social común ligado al desarrollo cultural.

En esta línea metodológica, aparece la figura del interventor comunitario cuya misión será ofrecer estrategias para que la comunidad se conciba como agente de cambio con recursos disponibles para el control de sus vidas y la resolución de problemas.

El acercamiento a los recursos supone una mayor implicación de los usuarios en la práctica del trabajo comunitario. Lejos de una actitud paternalista centrada en un modelo de déficit, desde el modelo de potenciación se consideran con los suficientes recursos y habilidades como para ayudar y ser ayudados. Las personas pasan a ser colaboradoras y el colaborador es un participante involucrado, así se genera el proceso de influencia mutua (Chavis).

\section{Ejemplo práctico}

Lola (nombre ficticio) es una mujer que vive con su marido y su hija de trece años en la localidad de Getafe. Su psiquiatra valora la derivación a recurso de centro de rehabilitación laboral, estando ya atendida por el Centro de Día de Getafe.

En los primeros momentos, el equipo pone el foco de atención en el proceso de evaluación de Lola a través de la asistencia a los diferentes talleres pre laborales y citas mantenidas con los profesionales del centro (psicóloga, maestros de taller, preparadora laboral, técnica de inserción laboral y terapeuta ocupacional).

En la elaboración de su Plan Individual de Recuperación Laboral (PIRL) en abril de 2019, los profesionales invitan a Lola a participar en su PIRL para acordar objetivos en el centro.

Se le ofrece libertad para elegir el formato de esta reunión, es decir, si desea o no citarse con todos los profesionales, facilidad en el horario y tiempo para conciliar con su rol de madre y ama de casa, expresarse con libertad en el caso de desacuerdo o planteamiento de nuevos objetivos, etc.

Tras esto, las necesidades, expectativas y objetivos en las diferentes áreas de intervención, fueron los siguientes: 


\begin{tabular}{|c|c|c|c|}
\hline ÁREAS & $\begin{array}{l}\text { NECESIDADES } \\
\text { Y EXPECTATIVAS }\end{array}$ & $\begin{array}{l}\text { OBJETIVOS } \\
\text { ESPECÍFICOS }\end{array}$ & $\begin{array}{l}\text { PROGRAMAS } \\
\text { ESPECÍFICOS }\end{array}$ \\
\hline Salud & $\begin{array}{l}\text { "Apoyo continuo por los } \\
\text { problemas de memoria" }\end{array}$ & $\begin{array}{l}\text { Evaluar las dificultades cognitivas. } \\
\text { Mejorar habilidades de } \\
\text { afrontamiento. }\end{array}$ & $\begin{array}{l}\text { Programa de afrontamiento. } \\
\text { Programa de atenciones } \\
\text { psicológicas. } \\
\text { Taller Felizmente }\end{array}$ \\
\hline Autonomía & $\begin{array}{l}\text { "Ayudar al principio para conocer } \\
\text { la ruta para ir a los sitios" } \\
\text { "Necesitaría dinero para el } \\
\text { transporte en entrevistas y para } \\
\text { empezar a trabajar" }\end{array}$ & $\begin{array}{l}\text { Apoyar los inicios de rutas para } \\
\text { aprender los recorridos. } \\
\text { Gestionar el proceso iniciado } \\
\text { en noviembre para una nueva } \\
\text { valoración del certificado de } \\
\text { discapacidad. } \\
\text { Buscar ayudas económicas en } \\
\text { general }\end{array}$ & $\begin{array}{l}\text { Programa de funcionamiento } \\
\text { psicosocial }\end{array}$ \\
\hline $\begin{array}{l}\text { Formativa, } \\
\text { laboral y legal }\end{array}$ & $\begin{array}{l}\text { "Encontrar un trabajo (en } \\
\text { horario de colegio de su hija): } \\
\text { tele operada, diseño gráfico, } \\
\text { acompañamiento, limpieza, etc." } \\
\text { "Cree que necesita aprender } \\
\text { las técnicas de búsqueda de } \\
\text { empleo" }\end{array}$ & $\begin{array}{l}\text { Aprender las técnicas de búsqueda } \\
\text { de empleo } \\
\text { Buscar trabajo en empleo protegido } \\
\text { Conocer las funciones y tareas de } \\
\text { diferentes áreas laborales. }\end{array}$ & $\begin{array}{l}\text { Programa de entrenamiento en } \\
\text { búsqueda activa de empleo. } \\
\text { Programa de orientación } \\
\text { vocacional }\end{array}$ \\
\hline Ajuste laboral & $\begin{array}{l}\text { Me gustaría tener un estilo más } \\
\text { profesional. }\end{array}$ & & Talleres pre laborales \\
\hline Red social y ocio & $\begin{array}{l}\text { "Me gustaría salir más y tener } \\
\text { más tiempo y tener más contacto } \\
\text { con compañeros del Centro de } \\
\text { Día, alguna amiga o gente del } \\
\text { CRL" }\end{array}$ & $\begin{array}{l}\text { Conocer a su marido, para entender } \\
\text { la dinámica familiar } \\
\text { Fortalecer los apoyos que pone en } \\
\text { marcha }\end{array}$ & Programa de familias \\
\hline
\end{tabular}

Como vemos, desde la acogida de Lola, se ha pretendido que tome decisiones y participe de manera activa, según los principios de empoderamiento.

Ahora, centrándonos en la intervención, desarrollaremos el caso según los niveles de análisis interdependientes para la potenciación de Zimmerman.

\section{Nivel psicológico:}

A partir del mes de mayo, momento de inicio de la intervención de diferentes profesionales, comenzamos a observar el bajo nivel de autoestima de Lola, evidenciándose en los mensajes que lanza hacia su persona de manera continuada. Se percibe con dificultades cognitivas, incapaz de ser funcional en su vida cotidiana y estructurar los tiempos de la actividad doméstica, durante los talleres pre laborales, se muestra nerviosa e interferida por pensamientos relacionados con la economía del hogar, su rol de madre y esposa.

En las coordinaciones mantenidas con el centro de día al que acude, nos indican las dificultades que están teniendo con Lola para trabajar las capacidades cognitivas, dado que prioriza constantemente la demanda de su entorno convivencial a ella misma y no es constante en la asistencia.
Desde el CRL, intentamos adaptar los tiempos que dedica a cada tarea comenzando por los autocuidados, pero le cuesta integrar y llevar a cabo las pautas ofrecidas. Por otro lado, mantiene la asistencia de manera intermitente a orientación vocacional individual, ajustando los horarios a sus necesidades. También tiene citas con preparadora laboral donde hacen entrenamiento en búsqueda activa de empleo, incidiendo en la parte de "entrevista" y valorando oportunidades laborales.

Comenzamos a dar apoyo para gestionar los ingresos en el hogar, la solicitud de prestaciones económicas y sugerencias para equilibrar su rol de madre, su papel de ama de casa y esposa.

Invitamos a Lola a mantener una cita con su marido para tratar toda esta parte y acepta, pero durante los meses venideros en los que intentamos contactar con él, en una ocasión se produce el encuentro con el marido, quien resta importancia a la supuesta mala gestión de la economía en casa y atribuye a Lola cualidades empobrecidas. Entre tanto, Lola nos comenta su preocupación por comportamientos extraños del marido y como este extrae dinero de la cuenta bancaria común y adquiere deudas y acuerdos con personas que ella no conoce. Esto hace que perciba inseguridad y desconfianza en torno a su marido y miedos por las consecuencias que pueda 
traer las acciones de su marido, sobre su hija y ella, convirtiéndose el hogar en un entorno hostil.

A finales de diciembre, Lola nos traslada su intención de divorciarse del marido pues "está arrastrándonos a mi hija y a mí". Declara esta intención a sus familiares que la apoyan en la decisión. Llega a entablar un encuentro con el abogado en enero, pero meses después y con la llegada del confinamiento, la idea decae y prefiere esperar a ver si su marido cambia.

El confinamiento interrumpe en parte, el proceso de potenciación y clínicamente empeora su situación, siendo apoyada por el equipo técnico a través de video llamadas, llamadas y mensajes de WhatsApp y por su psiquiatra. Durante este tiempo, acepta comenzar un curso de manipulador de alimentos, manejando la Tablet y el portátil de su hija. Al principio se muestra dubitativa "porque soy unas manazas y lo voy a romper" y le invitamos a pedir ayuda a la pequeña y así lo hace, resultando ser positivo pues acepta. No logra terminar la formación, pero, por otro lado, transmite que ha podido interactuar con su hija de otra manera, regulando su rol de madre en este sentido, no siendo ella quien ofrezca siempre los cuidados.

La primera cita presencial en el CRL llega en junio, tras la reevaluación de los objetivos y su incorporación a un puesto de empleo que detallamos en el nivel comunitario.

\section{Nivel organizacional:}

Lola posee buenas habilidades sociales y entabla con facilidad conversación con las compañeras del centro. Durante el mes de junio, le interesa colaborar en la compra, elaborando la lista y haciendo apuntes en una libreta, de los productos y el gasto acumulado. Al poco tiempo, abandona por los cuidados y preocupaciones provenientes de la falta de control de la problemática psicosocial (ámbito doméstico, relación familiar, economía del hogar, etc.).

Ya en octubre, se le ofrece acudir a un grupo de pintura donde se desenvuelve con arte en la materia y así se lo hacen ver los integrantes del grupo. Logra mantenerse hasta la situación de confinamiento, donde se muestra interesada por el grupo de mujeres que surge con el fin de dar apoyo mutuo, ejerciendo un rol activo, indicando las vivencias y actividades del día, animando a las compañeras, compartiendo fotos, etc.

\section{Nivel comunitario:}

Como habíamos acordado con ella, en mayo iniciamos la valoración de las prestaciones que pueden ayudarle a adquirir mayor colchón económico. Le informamos de la RAI, un ingreso destinado a personas con discapacidad parada de larga duración. Le apoyamos en los trámites y finalmente, a mediados de junio, comienza a percibir este ingreso. Hablamos entonces de la compatibilidad con un trabajo, pero Lola teme perder la prestación. Aun así, decide seguir adelante con el itinerario formativo- laboral para continuar creciendo.
Entre tanto, Lola nos informa que ha solicitado la reevaluación del certificado de discapacidad del $38 \%$ con la intención de obtener un $65 \%$. Su intención es optar a una incapacidad laboral que les conceda acceso a otras prestaciones económicas. Ella misma solicita a atención primaria la derivación al neurólogo. Más tarde, el neurocirujano le informa de la detección de unas manchas en la imagen del TAC que pudieran estar relacionadas con

su deterioro cognitivo. En paralelo, valoramos pasar una escala sencilla de detección del deterioro cognitivo donde el resultado no evidencia tal problemática.

Lola solicita acompañamiento para la valoración en el centro base, de su grado de discapacidad. Los dos profesionales que nos atienden, psicólogo y médico, concluyen que la situación clínica de Lola no ha variado. Lola alega su deterioro cognitivo y el informe del neurocirujano, pero ambos le refieren que no es concluyente para justificar un deterioro cognitivo y necesitan la evaluación de un neuropsicologo que argumente tal hecho.

Le dan mensajes de fortaleza, pues es una mujer capaz de llevar su casa, los cuidados de su hija y la asunción de un futuro empleo con las capacidades que preserva a pesar de su historial clínico, considerando que puede llegar a ser una mujer plenamente funcional.

En junio acude a la jornada de empleo de Esencial Jobs, empresa con la que surge una entrevista en limpieza que finalmente, no sale adelante.

En Julio, le informamos de la posibilidad de inscribirse al programa de formación para el empleo de Getafe llamado POEFE de ALEF. Valora solicitar el curso de "Limpieza de superficies y mobiliario en edificios y locales". También se inscribe y acude en diciembre a la entrevista del POEFE de Móstoles para el curso de "Auxiliar de Estética" pero no logra conseguir la plaza.

En septiembre, acude a una entrevista con una agencia de colocación que finalmente, no sale adelante.

En enero nos comenta que ha comenzado a informarse a través de la Casa de la Mujer de Getafe y un abogado, sobre cómo tramitar el divorcio con su marido.

En este mismo mes, CEE Manantial Integra nos ofrece unas prácticas no laborales (PNL) de limpieza de febrero a marzo, con la posibilidad de incorporarse a una bolsa de empleo. Se lo planteamos a Lola y traslada de nuevo su miedo a perder la RMI. Vemos con ella que es un riesgo a contemplar y le invitamos a no perder de vista la oportunidad para comprobar por sí misma, si dispone de competencias suficientes en la ejecución de las tareas derivadas del puesto de limpieza, en un entorno laboral. Decide seguir adelante con el proceso y le ayudamos a preparar la entrevista con Manantial.

La buena noticia llega días después, tras aceptar la candidatura de Lola en limpieza en una mini residencia de la zona sur de Madrid.

El primer día se pierde, pero se reubica y logra llegar. Se siente bien acogida por las diferentes personas que se encuentran en la mini residencia.

Al finalizar las prácticas y coordinarnos para ver los resultados, nos comentan que Lola ha superado satisfactoriamente las PNL y pasan a incorporar su perfil laboral, en la bolsa de empleo. 
Tras el confinamiento, en mayo se produce la llamada para trabajar en el mes de junio. El contrato dura unos días. Lola acoge bien la noticia pues conocía que era una IT y podría suceder. Valora que ha tenido algunos despistes y dudas durante el desempeño, pero siente que ha hecho bien su trabajo.

Amediados de junio, la CEE Manantial Integra, vuelve a ponerse en contacto con ella para ofrecerle trabajo en limpieza desde Julio hasta finales de septiembre. Acepta con ilusión esta nueva oportunidad.

Antes de que comience con su nuevo itinerario laboral, nos reunimos con ella para hacer seguimiento de sus objetivos y son observables los cambios:
Actualmente, continúa desarrollando sus labores de limpieza en la Mini residencia y seguimos acompañándola en su proceso de potenciación.

\section{Conclusiones}

Como podemos ver, las experiencias adquiridas en su entorno personal, en la formación y en el empleo, ha supuesto un proceso de cambio y transformación hacia una visión más amable y realista de sí misma. Las posibilidades contextuales han facilitado que Lola haya

\begin{tabular}{|c|c|c|c|c|}
\hline ÁREAS & $\begin{array}{l}\text { NECESIDADES Y } \\
\text { EXPECTATIVAS }\end{array}$ & $\begin{array}{l}\text { OBJETIVOS } \\
\text { ANTERIORES }\end{array}$ & $\begin{array}{c}\text { VALORACIÓN/CAMBIOS } \\
\text { QUE PERCIBE }\end{array}$ & $\begin{array}{l}\text { NUEVOS } \\
\text { OBJETIVOS }\end{array}$ \\
\hline Salud & $\begin{array}{l}\text { "Mejorar los } \\
\text { mensajes que me } \\
\text { doy" }\end{array}$ & $\begin{array}{l}\text { Mejorar habilidades } \\
\text { de afrontamiento. } \\
\text { Mejorar la toma de } \\
\text { decisiones }\end{array}$ & $\begin{array}{l}\text { He usado estrategias para } \\
\text { compensar mis supuestos "fallos } \\
\text { de memoria" }\end{array}$ & $\begin{array}{l}\text { Conseguir valoración por } \\
\text { neuropsicólogo. } \\
\text { Trabajar en un discurso más } \\
\text { amable conmigo misma. }\end{array}$ \\
\hline Autonomía & $\begin{array}{l}\text { "Recibir apoyo } \\
\text { puntual para } \\
\text { organizar recibos y } \\
\text { pagos" } \\
\text { "Bajar de peso" }\end{array}$ & $\begin{array}{l}\text { Mejorar la } \\
\text { organización diaria, } \\
\text { el cuidado de la } \\
\text { salud, bajar de peso. }\end{array}$ & $\begin{array}{l}\text { He dedicado gran parte del tiempo } \\
\text { a organizar a través de la agenda, } \\
\text { esto ha hecho que sea más lenta a } \\
\text { la hora de hacer otras actividades. }\end{array}$ & $\begin{array}{l}\text { Mejorar el uso de las nuevas } \\
\text { tecnologías. } \\
\text { Recibir apoyo puntual para } \\
\text { organizar recibos y pagos. } \\
\text { Llevar la casa entre todos. }\end{array}$ \\
\hline $\begin{array}{l}\text { Formativa } \\
\text { y laboral }\end{array}$ & & $\begin{array}{l}\text { Adquirir estrategias } \\
\text { de búsqueda de } \\
\text { empleo. } \\
\text { Formarse } \\
\text { Buscar su área } \\
\text { laboral }\end{array}$ & $\begin{array}{l}\text { He hecho una formación en } \\
\text { prácticas en limpieza y he } \\
\text { encontrado empleo. } \\
\text { Siempre he pensado que no me } \\
\text { gustaría, pero haciéndolo, me he } \\
\text { sentido a gusto, motivada y con } \\
\text { ganas de seguir. Me ha gustado } \\
\text { el ambiente laboral. Traslado ese } \\
\text { ánimo a casa. }\end{array}$ & $\begin{array}{l}\text { Aprender mejor el oficio. } \\
\text { Reducir el riesgo físico } \\
\text { que entraña hacer tareas } \\
\text { de limpieza. Ergonomía } \\
\text { postural laboral. } \\
\text { Mantener el puesto de } \\
\text { trabajo. }\end{array}$ \\
\hline $\begin{array}{l}\text { Ajuste } \\
\text { laboral }\end{array}$ & & $\begin{array}{l}\text { Conocer nuevas } \\
\text { habilidades laborales }\end{array}$ & & \\
\hline $\begin{array}{l}\text { Red social } \\
\text { y ocio }\end{array}$ & & $\begin{array}{l}\text { Fortalecer los apoyos } \\
\text { que pone en marcha }\end{array}$ & & $\begin{array}{l}\text { Mejorar la comunicación } \\
\text { interpersonal con amigos y } \\
\text { familia. } \\
\text { Tener tiempo para salir. }\end{array}$ \\
\hline
\end{tabular}

Como podemos ver, con las experiencias adquiridas en su entorno personal, en la formación y en el empleo, poco a poco Lola está logrando empoderarse y abrazar una figura más real y amable de su persona, proyectando nuevas metas de crecimiento. podido sopesar y liderar su toma de decisiones, siendo la protagonista de su recuperación e inserción laboral. Actualmente, continúa desarrollando sus labores de limpieza en la miniresidencia y seguimos acompañándola con una mirada de empoderamiento. 


\section{Documentos}

Achieving health equity: from root causes to fair outcomes. Commission on Social Determinants of Health. Interim statement. Geneva, World Health Organization, 2007 (http://whqlibdoc.who.int/publications/2007/interim_statement_eng.pdf, accessed 11 May 2009).

Convention on the Rights of Persons with Disabilities and its Optional Protocol. New York, United Nations, 2006 (http://www. un.org/disabilities/default.asp?id=150, accessed 11 May 2009).

CSDH. Closing the gap in a generation: health equity through action on the social determinants of health. Final Report of the Commission on Social Determinants of Health. Geneva, World Health Organization, 2008.

Declaration of Alma-Ata. Copenhagen, WHO Regional Office for Europe, 1978 (http://www.euro.who.int/AboutWHO/ Policy/20010827_1, accessed 11 May 2009).

Empowerment and mental health advocacy. Briefing paper for the WHO European Ministerial Conference on Mental Health: Facing the Challenges, Building Solutions. Copenhagen, World Health Organization, 2005.

European Pact for Mental Health and Well-being. Brussels, European Commission, 2008 (http://ec.europa.eu/health/ph_determinants/ life_style/mental/docs/pact_en.pdf, accessed 11 May 2009.

Gaining Health. The European Strategy for the Prevention and Control of Noncommunicable Diseases. Copenhagen, WHO Regional Office for Europe, 2006 (http://www.euro.who.int/InformationSources/Publications/Catalogue/20061003_1, accessed 11 May 2009).

Mental Health Action Plan for Europe. Copenhagen WHO Regional Office for Europe (http://www.euro.who.int/Document/ MNH/edoc07.pdf, accessed 11 May 2009).

Mental Health Declaration for Europe. Copenhagen, WHO Regional Office for Europe (http://www.euro.who.int/document/mnh/ edoc06.pdf, accessed 11 May 2009).

Ottawa Charter for Health Promotion. Copenhagen, WHO Regional Office for Europe, 1986(http://www.euro.who.int/AboutWHO/ Policy/20010827_2, accessed 11 May 2009).

Policies and practices for mental health in Europe. Meeting the challenges. Copenhagen, WHO Regional Office for Europe, 2008 (http://www.euro.who.int/InformationSources/Publications/Catalogue/20081009_1, accessed 11 May 2009).

\section{Bibliografía}

Bakker, L., y Van Brakel, W. (2012). "Empowerment assessment tolos in people with disabilities in developing countries", Lepr Rev, 83 (2): 129-153.

Fernández de Sevilla Otero, J. P., y San Pío Tendero, M. J. (2014). Guía PARTISAM: Promoción de la participación y autonomía en Salud Mental. Madrid: Asociación Española de Neuropsiquiatría.

Fitzimmons, S y Fuller, R. (2002). "Empowerment and its implications for clinical practice", Mental Health: a Review Journal of Mental Health, 5: 481-499.

Israel, B.A. et al. (1994). "Health education and community empowerment: conceptualizing and measuring perceptions of individual, organizational, and community control", Health Education Quarterly, 21:149-170.

Loss J, y Wiese M. (2008). "Evaluation von Empowerment - Perspektiven und Konzepte von Gesundheitsförderung”, Ergebnisse einer qualitativen Studie in Australien. 70: 755-763.

Musitu, G. \& Buelga, S. (2004). “Desarrollo Comunitario y Potenciación”. En Musitu, G., Herrero, J., Cantera, L., y Montenegro, M. (eds.), Introducción a la Psicología Comunitaria. Barcelona: UOC, pp. 167-195.

Pick, S., Sirkin, J., Ortega, I., Osorio, P., Martínez, R., Xocolotzin, U., Givaudan, M. (2007). “Escala para medir agencia personal y empoderamiento", Journal of Psychology, 41 (3): 295-304.

Rivera, N., y Gallardo, C. (2005). Grupos de ayuda mutua y asociaciones relacionadas con la salud: Claves de un nuevo modelo socio-sanitario. Madrid: Plaza y Valdés.

Rogers, E.S. et al. (1997). "Consumer-constructed scale to measure empowerment among users of mental health services", Psychiatric Services, 48 (8):1042-1047.

Vázquez-Barquero, J.L. et. al. (2000). "Disability assessment Schedule II: Initial phase of development and pilot study", Actas de Especialidad Psiquiátrica, 8 (2): 77-87.

Wallerstein N. (2006). What is the evidence on effectiveness of empowerment to improve health? Copenhagen: WHO Regional Office for Europe. 
